\title{
SIZE EFFECT FOR SAMPLES WITH BLUNT AND SHARP NOTCHES USING LINEAR COHESIVE CRACK LAW
}

\section{GIOVANNI DI LUZIO* ${ }^{*}$ and GIANLUCA CUSATIS ${ }^{\dagger}$}

\author{
*Politecnico di Milano \\ Milan, Italy \\ e-mail: giovanni.diluzio@polimi.it \\ ${ }^{\dagger}$ Northwestern University \\ Evanston, IL, USA \\ e-mail: g-cusatis@ northwestern.edu
}

Key words: Cohesive Fracture, Cohesive Crack Model, Size Effect, Quasi-brittle Materials

\begin{abstract}
The object of this work is to provide a more general formulation of the cohesive size effect curve to include structural configurations with blunt and sharp notches. An extensive campaign of accurate numerical simulations, based on the cohesive crack model, is performed to compute the Generalized Cohesive Size Effect Curves (GCSEC) for typical test configurations featuring both sharp and blunt notches. The results are analyzed with reference to the classical Bažant's Size Effect Law (SEL) to investigate the relationship between GCSEC and SEL. This analysis shows that as specimen size tends to infinity, the SEL represents the asymptote of the GCSEC in the case of sharp notches, and that the SEL parameter known as the effective fracture process zone length is a material property, which can be expressed as a function of the Cohesive Crack Law (CCL) parameters. For blunt notches, however, the nominal strength of infinite size samples tends to a horizontal asymptote corresponding to the elastic limit. It is shown that the two results are not in contradiction because the effective fracture process zone length tends to zero as the radius of curvature tends to zero.
\end{abstract}

\section{INTRODUCTION}

The size effect is a typically phenomenon displaced by quasi-brittle materials, which present the structural strength, $\sigma_{N}$, depending on the structural size $D$. The structural strength is defined as a normalized measure of the load carrying capacity (peak load) respect to the structural size, see [4]. Let us consider only structures with positive geometries, i.e. with dimensionless energy release rate that increases as the normalized crack length increases. Using the cohesive crack model, the size effect for mode I fracture can be expressed using the following equation $[3,4,7]$ :

$$
\left(\frac{f_{t}^{\prime}}{\sigma_{N}}\right)^{2}=\Phi\left(D / l_{c h}\right)
$$

where $l_{c h}=E G_{F} / f_{t}^{\prime 2}$ is Hillerborg's characteristic length [2], $f_{t}^{\prime}=$ tensile strength, $E=$ Young's modulus, and $G_{F}=$ fracture energy. The size effect relationship represented symbolically by Eq. 1 is refereed as Cohesive Size Effect Curve (CSEC) and some authors [1,3] proposed analytical expressions of the function $\Phi$ by fitting the results of numerical simulations of cohesive crack propagation in geometrically similar samples of increasing size considering only the case of sharp notches.

In [1] considering five different structural configurations of sharp-notched specimens, they verified that (1) for small sizes the CSEC tends to the plastic limit; (2) the CSEC curves feature a straight line asymptotes which is inde- 
pendent of structural geometry, boundary conditions, and loading configurations when normalized by the Linear Elastic Fracture Mechanics (LEFM) dimensionless energy release parameters, $g_{0}$ and $g_{0}^{\prime}$ [4] for each particular case. They showed also that such asymptote coincides with Bažant's Size Effect Law (SEL) [5], which can be expressed as:

$$
\frac{1}{g_{0}^{\prime}}\left(\frac{f_{t}^{\prime}}{\sigma_{N}}\right)^{2}=\frac{g_{0} D}{g_{0}^{\prime} l_{c h}}+\frac{c_{F}}{l_{c h}}
$$

where $c_{F}$ is the so called Effective Fracture Process Zone Length (EFPZL)and for $c_{F}=0$ the SEL coincides with the size effect provided by LEFM. In [1] it was also shown that (1) the $c_{F} / l_{c h}$ ratio is independent of geometrical and loading configurations and it is equal to 0.44 for a linear CCL demonstrating that the EFPZL is a material property as it is assumed in typical SEL derivations; (2) the Fracture Process Zone (FPZ) length, i.e. the length of the ligament portion with cohesive stresses, and notch tip cohesive stress converge for increasing size to the constant values of $0.71 l_{c h}$ (for linear softening) and zero, respectively, regardless of the structural configuration.

The objective of this study, which is presented in more details in [8], is to provide a more general formulation of the cohesive size effect curve to include structural configurations with blunt notches. Such formulation, entitled Generalized Cohesive Size Effect Curve (GCSEC) is derived by fitting accurate numerical simulations of size effect.

\section{NUMERICAL SIMULATIONS WITH COHESIVE CRACK LAW}

Two different structural configurations are considered: the direct tension on center crack panel (CCP) and the three-point bending (TPB) with a span/depth ratio $S / D=3$. The structural strength of these configurations are $\sigma_{N}=$ $P_{u} / D t$ for CCP and $\sigma_{N}=1.5(S / D) P_{u} / D t$, for TPB, where $P_{u}=$ peak load, $D=$ panel depth, $t=$ panel thickness, and $S=$ beam span [4].

The numerical simulations were performed by using standard Finite Element Method
(FEM) techniques. The bulk of the discretization (Fig. 1b) was modeled with eight-node, elastic, iso-parametric elements [9], and the crack line was modeled with cohesive interface elements [10] governed by a linear CCL.

a)

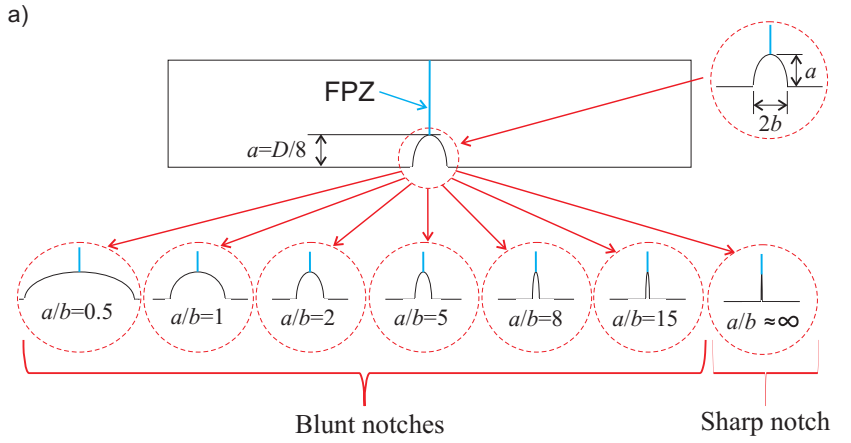

b)

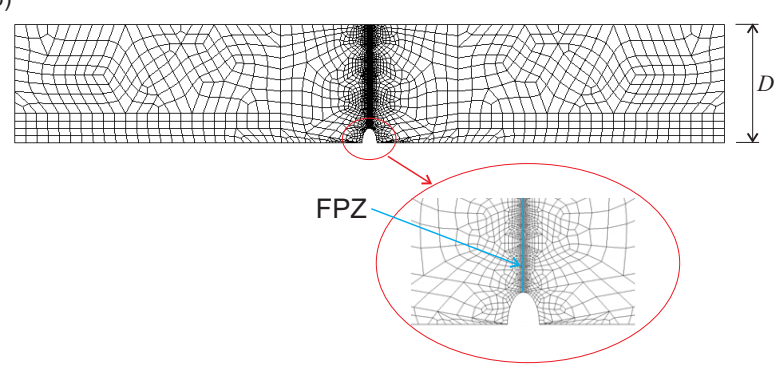

Figure 1: Structural configuration: (a) Typical semielliptical notch geometries used in the simulations. (b) Example of the FE mesh of the simulated specimens including zoom-in of the notch tip to show the mesh refinement required to capture correctly the stress concentration.

Numerical problems can arise when a stress singularity exists, for example, at the tip of a sharp notch. However, different situation arises when the notch is not perfectly sharp and features a small curvature radius. In this case, the stress is not infinite anymore, the stress singularity disappears and a finite stress concentration takes place. A stress concentration gives similar numerical challenges as stress singularities but the convergence is guaranteed provided that the mesh is sufficiently refined. The level of refinement depends on the radius of curvature of the notch: the smaller it is, the more refined the mesh must be.

Theoretically, to overcome the problems inherent in cohesive crack simulations with sharp notches one could simulate the peak load be- 
havior as the limit of elliptical notches as the elliptical aspect ratio $a / b$ (ratio of the longerto-shorter dimensions), tends to infinity (Fig. 17). While theoretically correct, this approach incurs also in numerical difficulties for high values $a / b$. Numerically, the best numerical response in presence of sharp notches can be obtained by modeling notches of small finite width, $b$, and a semi-circular tip (Fig. 1a, bottom right). The notch width, $b$, has to be kept constant and not to be scaled with specimen size. Although this violates the geometrical similarity of the specimens, it was shown [1] to provide an excellent approximation of sharp notch cohesive behavior if $b / l_{c h} \ll 1$.

To examine the change in size effect behavior with the presence of geometrical similar specimens, structural geometries with elliptic notches have been considered. The notch geometry is characterized by an semi-elliptical curve with the two semi-axis which represent the the half-notch opening, $b$, and the notch depth, $a$, see Fig. 11a. The perfect geometrical similarity is obtained by considering the structural response of geometries, in which the notch opening, $b$, of the elliptical notch is also scaled with the size. In particular, for the two different considered geometries constant and different value of the ratio $a / b$ were chosen (see Fig. 1 1a). In this case the notch has generally a blunt shape and the sharp notch is only obtained has limit case when the notch opening tends to zero.

For the reasons discussed above the resolution of finite element meshes utilized to simulate the crack propagation with the cohesive crack model must be very fine especially in the region close to the notch tip as shown in Fig. 1b. In all the simulations, the size of the interface elements ahead of the notch tip and for the entire length of the Fracture Process Zone (FPZ), was not scaled with the specimen size and it was kept within the relatively small range of 0.5-1.0 mm (Fig. [1 b).
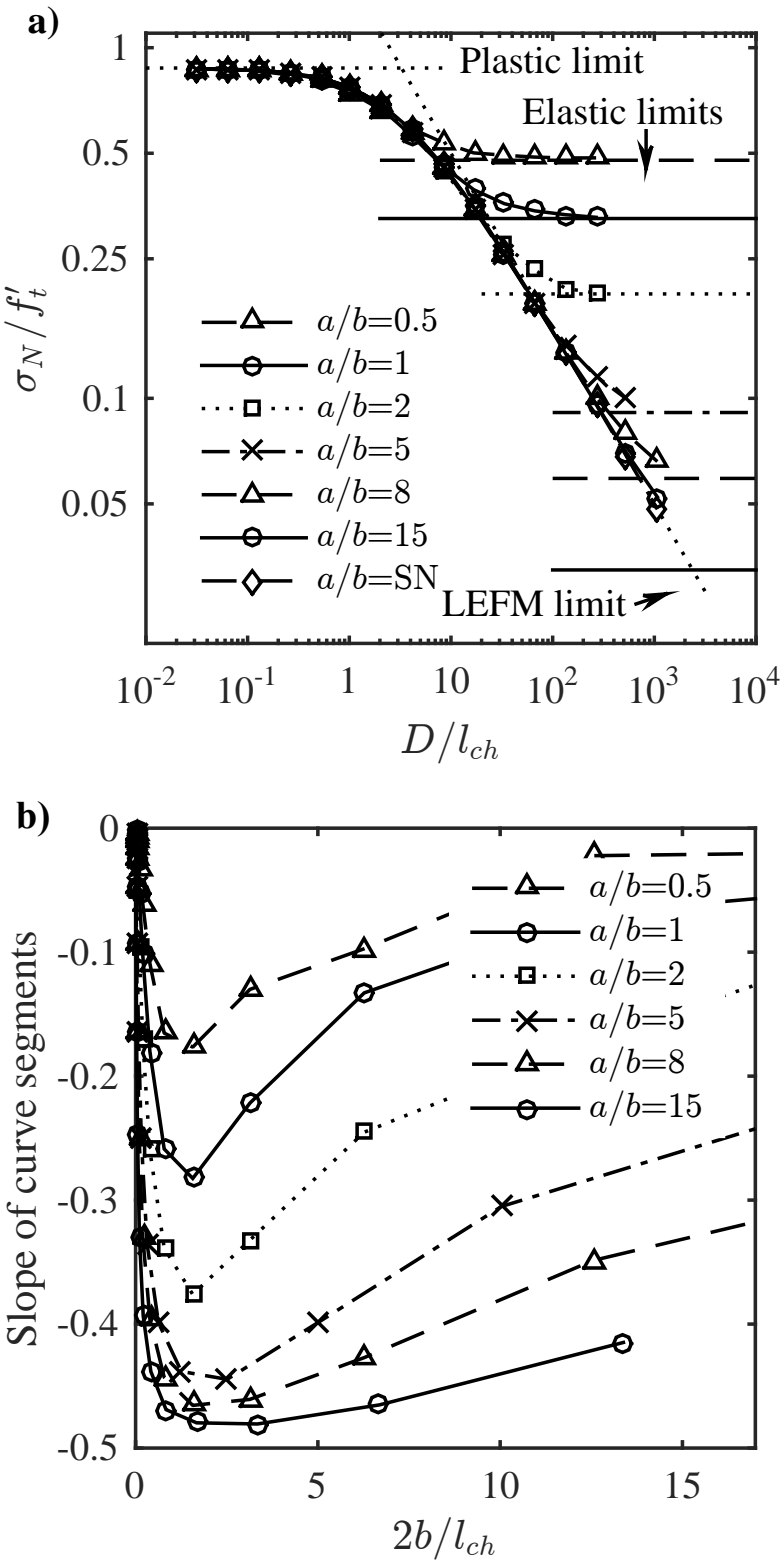

Figure 2: CCP Configuration: (a) size effect curves for elliptically notched geometries, (b) slope of size effect curves versus normalized width of elliptical notch.

The peak load was determined for sixteen sizes from $D=5 \mathrm{~mm}$, doubling 15 times up to $D=163,840 \mathrm{~mm}$ for different aspect ratio. In all the geometries the ratio between the notch depth $a$, and the panel depth $D$, was kept constant: $\alpha=a / D=0.125$. The numerical analyses were performed with a linear CCL and the following material properties: $E=24,000$ $\mathrm{N} / \mathrm{mm}^{2}, G_{F}=0.05 \mathrm{~N} / \mathrm{mm}, f_{t}^{\prime}=2.8 \mathrm{~N} / \mathrm{mm}^{2}$, corresponding to $l_{c h}=153 \mathrm{~mm}$. It is worth observing that the size effect curve does not de- 
pend on the specific material properties used in the calculations.
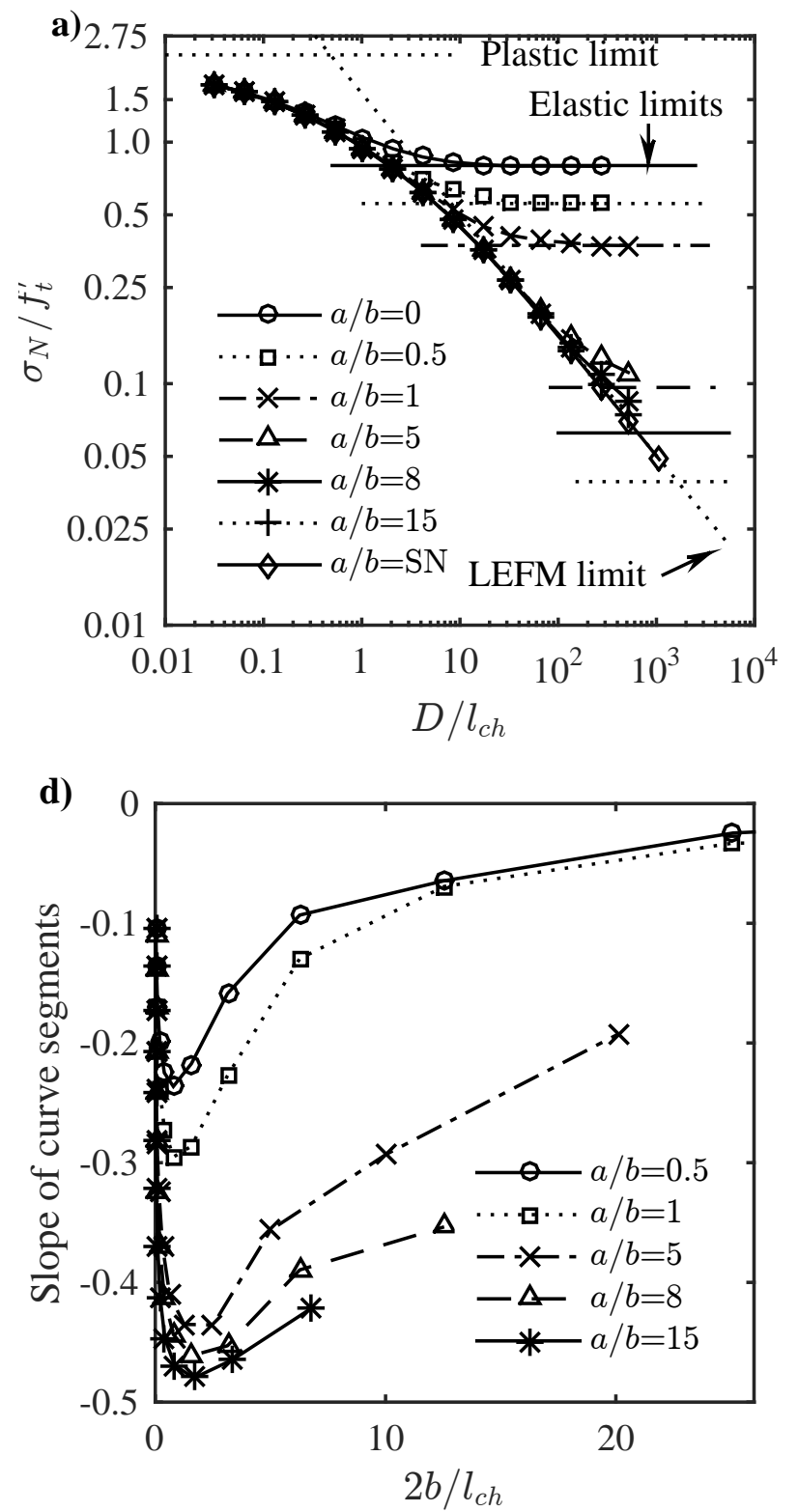

Figure 3: TPB Configuration: (a) size effect curves for elliptically notched geometries, (b) slope of size effect curves versus normalized width of elliptical notch.

The calculated size effect curves are shown in a log-log plot in Fig. 2a and Fig. 3a for the CCP and TPB configurations, respectively. In addition to the size effect curves, the plastic limit, the LEFM limit, and the elastic limits for each notch aspect ratio, are shown in the plots. The elastic limit is defined as a stress state where the normal stress at the tip of the notch first reaches the tensile strength, $f_{t}^{\prime}$, and it corresponds to the cohesive crack initiation. The nominal stress associated with the elastic limit can be calculated using the stress concentration factors at the notch tip: $\sigma_{x x}^{t i p}=k \sigma_{N}$. Since at the elastic limit $\sigma_{x x}^{t i p}=f_{t}^{\prime}$, the elastic nominal stress is $\sigma_{N e}=f_{t}^{\prime} / k$ and its normalized value is $\sigma_{N e} / f_{t}^{\prime}=1 / k$.

For the tip of an elliptical hole in a CCP geometry, the stress concentration factor $k$ in a finite-width plate and for $0.5 \leq a / b \leq 10.0$ can be calculated approximately with the following equations [11]:

$$
\begin{array}{r}
C_{1}=+1.00+2.00(a / b) ; \\
C_{2}=-0.351+0.21 \sqrt{a / b}-2.483(a / b) ; \\
C_{3}=+3.621-5.183 \sqrt{a / b}+4.494(a / b) ; \\
C_{4}=-2.27+5.204 \sqrt{a / b}-4.011(a / b) .
\end{array}
$$

For the $a / b$ equal to $0.5,1.0,2.0,5.0,8.0$, and $15 k$ equals $2.094,3.071,5.038,10.976,16.933$ and 30.862, respectively, and the corresponding normalized elastic limit, $1 / k$, is $0.4775,0.3257$, $0.1985,0.0911,0.0591$ and 0.0324 . For the unnotched case, $a / b=0, k=1$. It is worth reminding that in the $\mathrm{CCP}$ geometry with infinite plate the stress concentration factor $k$ is $k=1.0+2.0(a / b)$. These analytical values were found to match very well the ones computed numerically.

For the TPB configuration the normalized nominal stress at the elastic limit were obtained numerically as $0.5568,0.3736,0.0964$, and 0.0625 for notch aspect ratios of $0.5,1.0$, 5.0, and 8.0, respectively. The modulus of rupture, for geometry with aspect ratio equals to 0 , is expected be to be larger than the tensile strength because the stress distribution [13]. According to [12] the peak stress is given by $\sigma_{\text {tip }}^{\text {ela }}=\sigma_{N} / \beta$ with $\beta=(1-0.1773 D / S)^{-1}$. For the geometry considered in this study one has $\beta=[1-0.1773(1-\alpha) / 3]^{-1}=1.054$. At the elastic limit, $\sigma_{\text {tip }}^{\text {ela }}=f_{t}^{\prime}$, one obtains $\sigma_{N e}=\beta(1-\alpha)^{2} f_{t}^{\prime}=0.807 f_{t}^{\prime}$. As expected, the elastic limits are size-independent (they only depend on $\alpha$ and $a / b$ ) and they are plotted as horizontal straight lines in Fig. $2 \mathrm{a}$ and $3 \mathrm{a}$, for 
the TPB and CCP configurations, respectively. Similar results were obtained in [6] using a different approach.

The same plots $(2 \mathrm{a}$ and $3 \mathrm{a})$ show the plastic limits as horizontal lines. Since the plastic limit is independent on the notch aspect ratio one single horizontal straight line is common to all notch aspect ratios. The plastic limit is defined as a stress state where the entire crosssection ahead of, and collinear with, the original notch is subject to a uniform stress state equal to the tensile strength, $f_{t}^{\prime}$. For the CCP configuration, the nominal stress associated with the plastic limit can be calculated by considering the free-body diagram of one half of the panel with a uniform distribution of stress along the central line and by equating the forces acting at each end: $\sigma_{N} t D=f_{t}^{\prime}(1-\alpha) t D$. Substituting $\sigma_{N p}$ for $\sigma_{N}$ and rearranging leads to $\sigma_{N p}=f_{t}^{\prime}(1-\alpha)$, and so the normalized nominal stress associated with the plastic limit is $(1-\alpha)=0.875$. The plastic limit behavior for the TPB configuration is less intuitive to calculate [4, 14]. In the limit of $D \rightarrow 0$ the central cross section with the cohesive crack law consists of two parts: one of finite depth under tension with a perfectly plastic uniform distribution of stress and a concentrated force in the compressed zone which shrinks to a point. For TPB specimen, at the zero size limit, one has $\sigma=f_{t}^{\prime}=$ uniform distribution through the entire ligament except a concentrated compression force $F_{c}=t D(1-\alpha) f_{t}^{\prime}$ acting on the compression side. In this situation, the bending moment in the notched cross section is $M=0.5 t D^{2}(1-\alpha)^{2} f_{t}^{\prime}$, the applied load is $P_{\max }=4 M / S$, and the plastic limit results in $\sigma_{N u}=3(1-\alpha)^{2} f_{t}^{\prime}$. Consequently, the normalized nominal stress at the plastic limit is $3(1-\alpha)^{2}=2.2969$.

Figs. $2 \mathrm{a}$ and $3 \mathrm{a}$ report also the LEFM size effect limit as a straight line with a slope of $-1 / 2$ in the log-log scale.

In Fig. 2all all of the calculated size effect curves for the CCP configuration tend to the plastic limit for $D \rightarrow 0$. As the sample size increases, they deviate from the plastic limit with slopes that become increasingly negative. In ad- dition, each curve has an inflection point where the slope begins to become less negative and tends back towards zero. The inflection points of the Fig. $2 \mathrm{a}$ curves correspond to the minimum slope values in the Fig. $2 \mathrm{~b}$ plots. Up to their individual inflection points, all curves in Fig. 2a are essentially coincident, and this "common" curve tends, for large enough $a / b$ values, to the LEFM limit. Past their inflection points, each curve departs from this common curve, and as $D \rightarrow \infty$, the size effect behavior tends to the corresponding elastic limit. The slopes of the Fig. 2 a curve segments were plotted as a function of the normalized elliptical notch width, $2 b / l_{c h}$, and this is shown in Fig. 2p. In this figure, one can see that the minimum value of slope, which corresponds to the inflection point in the associated size effect curve, occurs at $2 b / l_{c h} \approx 2$. Based on these observations it is clear that the size effect behavior for arbitrary $a / b$ begins an asymptotic approach towards the LEFM limit, on a size effect path common to all $a / b$. However, as the elliptical notch width, $2 b$, reaches an approximate value of $2 l_{c h}$, the size effect behavior inflects away from the LEFM limit. As $a / b$ tends to infinity (i.e. a sharp-notched geometry), $b \approx 0$ for all $D$, and so the size effect behavior never deflects from the common curve. This is also consistent with the fact that for a sharp notch the stress is singular and the elastic limit is zero, making the horizontal asymptote in the log-log plot to be located at $\infty$.

Similarly to the CCP geometry also the TBP geometry displays the same behavior for blunt notches, i.e. for $a / b=$ constant. Fig. 3a shows all of the calculated size effect curves which tend to the plastic limit for $D \rightarrow 0$ and to the corresponding elastic limit for $D \rightarrow \infty$. Only the size effect curve for sharp-notched specimens approaches asymptotically towards the LEFM limit, see Figs. 3p. As it can be seen in Fig. $2 \mathrm{p}$ the minimum value of slope, which corresponds to the inflection point in the associated size effect curve, occurs again at $2 b / l_{c h} \approx 2$.

The specimens with blunt notch with different value of $a / b$ have a completely differ- 
ent asymptotic behavior for $D \rightarrow \infty$. This is also confirmed by the length of the FPZ and the stress at the notch tip at peak load. As a matter of fact, the size of the FPZ at peak load shrinks and, for very large sizes, becomes almost a point. In addition, the stress at the notch tip tends to the tensile strength, $f_{t}^{\prime}$. As a consequence, for specimens with blunt notch the size of the FPZ at peak load is no more a material property not even for large sizes in contrast to the case for sharp notches. According to the literature, the size of the FPZ at peak load, for very large sizes, should be a material property [4]. However, the obtained results seem to contradict that principle: the curves for different values of $a / b$ appear to be approaching different asymptotes. On the other hand, the specimens with a sharp notch clearly have an asymptote of the size of the FPZ at peak load for $D \rightarrow \infty$ and the notch tip stress is closest to the fullysoftened condition.

\section{GCSEC FOR A LINEAR COHESIVE CRACK LAW}

An approximated analytical CSEC formula for TPB geometries with sharp notch was proposed in [1], inspired by the pioneering work of Planas [4], by interpolation of numerical results obtained with a linear cohesive law. That analytical expression was designed to match both the small-size (plastic limit) and large-size (asymptotic) behaviors and has the following expression

$$
\begin{aligned}
\frac{f_{t}^{\prime 2}}{g_{0}^{\prime} \sigma_{N u}^{2}}=\frac{g_{0} D}{g_{0}^{\prime} l_{c h}}+ & \left(1+11 \sqrt{\frac{g_{0} D}{g_{0}^{\prime} l_{c h}}}\right) \\
& \left(\beta_{0}+25 \sqrt{\frac{g_{0} D}{g_{0}^{\prime} l_{c h}}}\right)^{-1}
\end{aligned}
$$

where $\beta_{0}=9(1-\alpha)^{4} g_{0}^{\prime}$ and the associated SEL is given in the Eq. 2 .

We here propose a generalization of the expression in Eq. 7 to include the size effect of structures with both sharp and blunt notches. The new analytical formula, entitled Generalized Cohesive Size Effect Curve (GCSEC) is obtained by the interpolation of numerical results obtained with the adoption of a linear cohesive law. Keeping in mind, as showed in the previous section, that for large size $(D \rightarrow \infty)$ the GCSEC formula must tends to the Bažant's SEL for sharp notches and to a horizontal asymptote, the elastic limit, for blunt notches. In addition, this analytical expression is formulated in a such way to match the plastic limit for small sizes.

The GCSEC can be expressed by the following formula

$$
\begin{aligned}
\sigma_{N u}=f_{t}^{\prime} & \left(g_{0} \frac{D}{l_{c h}}+g_{0}^{\prime} \frac{1+11\left(\frac{g_{0} D}{g_{0}^{\prime} l_{c h}}\right)^{n}}{\beta_{p}^{2} g_{0}^{\prime}+25\left(\frac{g_{0} D}{g_{0}^{\prime} l_{c h}}\right)^{n}}\right)^{-1 / 2} \\
& \left(g_{0} \beta_{e}^{2} \frac{D}{l_{c h}}+\frac{1}{\left(1+g_{0} \beta_{e}^{2} \frac{D}{l_{c h}}\right)^{m}}\right)^{1 / 2}
\end{aligned}
$$

Alternatively, the GCSEC can be expressed similarly to CSEC in Eq. 7 as

$$
\begin{aligned}
\frac{f_{t}^{\prime 2}}{g_{0}^{\prime} \sigma_{N u}^{2}}= & \left(\frac{g_{0} D}{g_{0}^{\prime} l_{c h}}+\frac{1+11\left(\frac{g_{0} D}{g_{0}^{\prime} l_{c h}}\right)^{n}}{\beta_{p}^{2} g_{0}^{\prime}+25\left(\frac{g_{0} D}{g_{0}^{\prime} l_{c h}}\right)^{n}}\right) \\
& \left(g_{0} \beta_{e}^{2} \frac{D}{l_{c h}}+\frac{1}{\left(1+g_{0} \beta_{e}^{2} \frac{D}{l_{c h}}\right)^{m}}\right)^{-1}
\end{aligned}
$$

In both Eq. 8 and Eq. 9. $\beta_{e} f_{t}^{\prime}$ and $\beta_{p} f_{t}^{\prime}$ are the elastic limit and the plastic limit nominal stresses, respectively; the parameters $n$ and $m$ depend on the geometry.

The GCSEC function is plotted in Fig. 4 for CCP geometry and in Fig. 5 for TPB geometry. The best fitting of the numerical data sets was obtained with $n=0.45 m=0.7$ and $n=2$, $m=0.9$ for $\mathrm{CCP}$ and TPB configuration, respectively. Fig. $4 \mathrm{a}$ for CCP geometry and in Fig. 5 a for TPB geometry show clearly that the size effect curves merge in a single curve for different notch shape ratios $a / b$ and for $D / l_{c h}$ smaller than 1. In Fig. $4 \mathrm{p}$ and in Fig. 5p the GCSEC and numerical data are plotted in the parametric space given by $X=g_{0} D /\left(g_{0}^{\prime} l_{c h}\right)$ and $Y=f_{t}^{\prime 2} /\left(g_{0}^{\prime} \sigma_{N}^{2}\right)$ showing again a good fit. 
In the small size range (Fig. 4 for $\mathrm{CCP}$ geometry and in Fig. 5 for TPB geometry), the behavior is shown to be different for the two structural configurations. However, the GCSEC formula is capable of capturing the two different asymptotic behavior and value, which differ from the Bažant's SEL.
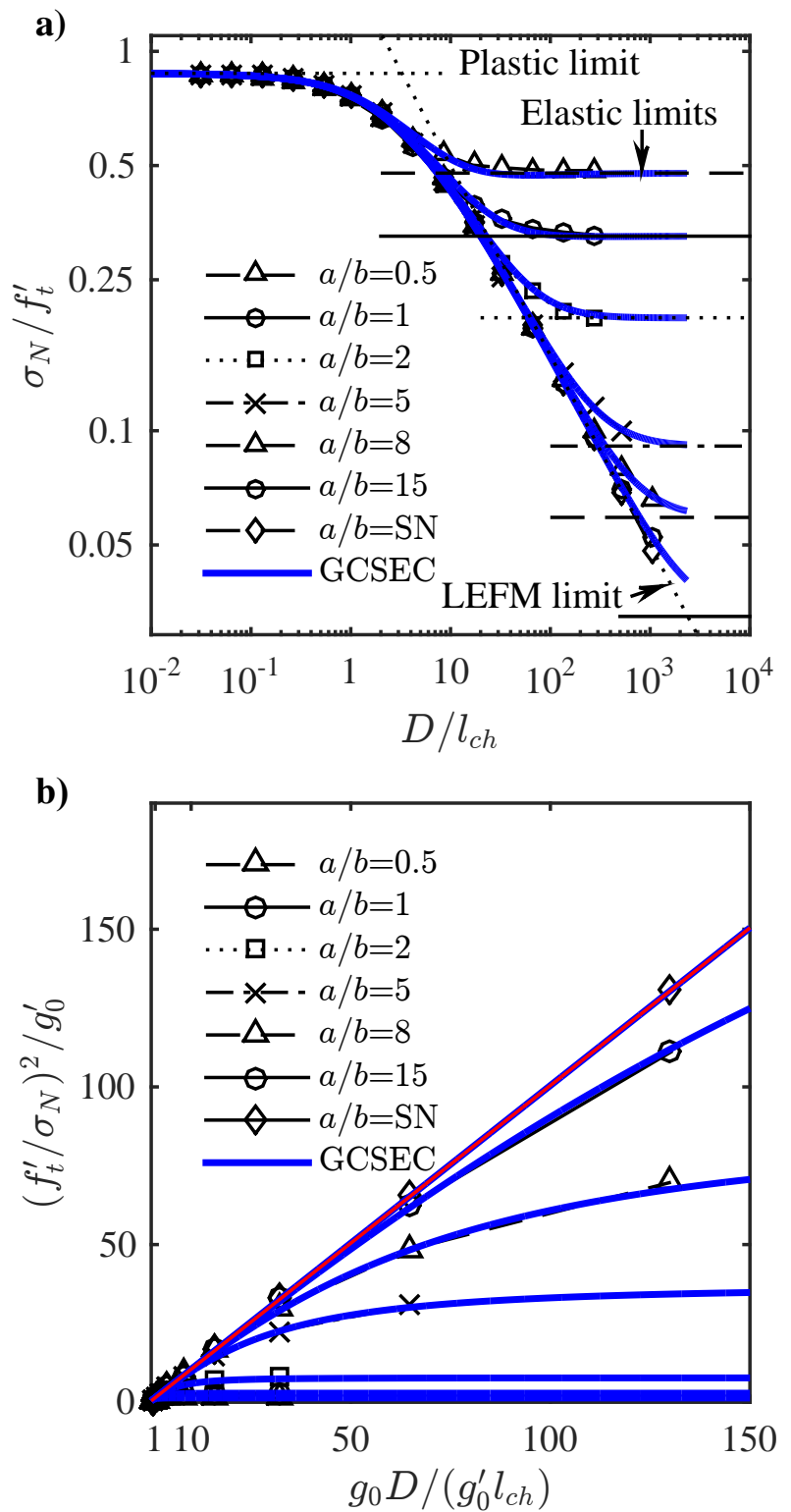

Figure 4: CCP Configuration: a) size effect curves for elliptically notched geometries; b) slope of size effect curves versus normalized size; c) slope of size effect curves versus normalized width of elliptical notch; d) size effect curve for constant-width notch geometry.

In the case of sharp notch, i.e. $a / b \rightarrow \infty$, the LEFM gives an elastic limit $\sigma_{N e}=0$, and so $\beta_{e}=0$, therefore the GCSEC function in Eq. 8 has the following expression

$$
\begin{gathered}
\sigma_{N u}=f_{t}^{\prime}\left(g_{0} \frac{D}{l_{c h}}+\right. \\
\left.g_{0}^{\prime} \frac{1+11\left(\frac{g_{0} D}{g_{0}^{\prime} l_{c h}}\right)^{n}}{\beta_{p}^{2} g_{0}^{\prime}+25\left(\frac{g_{0} D}{g_{0}^{\prime} l_{c h}}\right)^{n}}\right)^{-1 / 2}
\end{gathered}
$$

For $D \rightarrow \infty$, the previous expression, Eq. 10, tends to the SEL, Eq. 2. This means that the GCSEC formula for sharp notches has a large-size (asymptotic) behavior coinciding with Bažant's SEL. At the same time, for $D \rightarrow$ 0 , the nominal strength, $\sigma_{N}$, in Eq. 10 , tends to the plastic limit, i.e. $f_{t}^{\prime} \beta_{p}$.

In the case of blunt notch, for increasing size $D$ and arbitrary $a / b, \sigma_{N}$ begins an asymptotic approach towards the LEFM limit with a common path common to all $a / b$. However, as the elliptical notch width, $b$, reaches an approximate value of $l_{c h}$, the size effect behavior inflects away from the LEFM limit with a deviation of the GCSEC formula from the SEL to an horizontal asymptote $\left(\beta_{e} \neq 0\right)$ which depends on the actual values of $a / b$ and $\alpha$. For $D \rightarrow \infty$ the the asymptotic value of Eq. 8 is

$$
\begin{array}{r}
\lim _{D \rightarrow \infty} \sigma_{N u}=f_{t}^{\prime}\left(g_{0} \frac{D}{l_{c h}}+\frac{11}{25} g_{0}^{\prime}\right)^{-1 / 2} \\
\left(g_{0} \beta_{e}^{2} \frac{D}{l_{c h}}\right)^{1 / 2}=f_{t}^{\prime}\left(\beta_{e}^{2}\right)^{1 / 2}=f_{t}^{\prime} \beta_{e}
\end{array}
$$

We should remark that the normalized energy release rate, $g_{0}$, and its derivative, $g_{0}^{\prime}$, required for the blunt notch case are the LEFM reference values and they are calculated for the sharp notch case with the same $\alpha$, as for the GCSEC plotted in 4 for CCP geometry and in Fig. 5 for TPB geometry.

The case of failure that occurs at crack initiation from a smooth surface, i.e. no notch, is also considered in the GCSEC formula as a limit case when $a / b \rightarrow 0$. This case is taken into account in the Eq. 8 with the appropriate value of the elastic limit $\beta_{e}$. The asymptotic behavior for $D \rightarrow \infty$ of the GCSEC is the same 
of Eq. 11. For $D \rightarrow 0$, the nominal strength, $\sigma_{N}$, in Eq. 8, tends to the plastic limit, i.e. $f_{t}^{\prime} \beta_{p}$, like in the case of sharp notch.
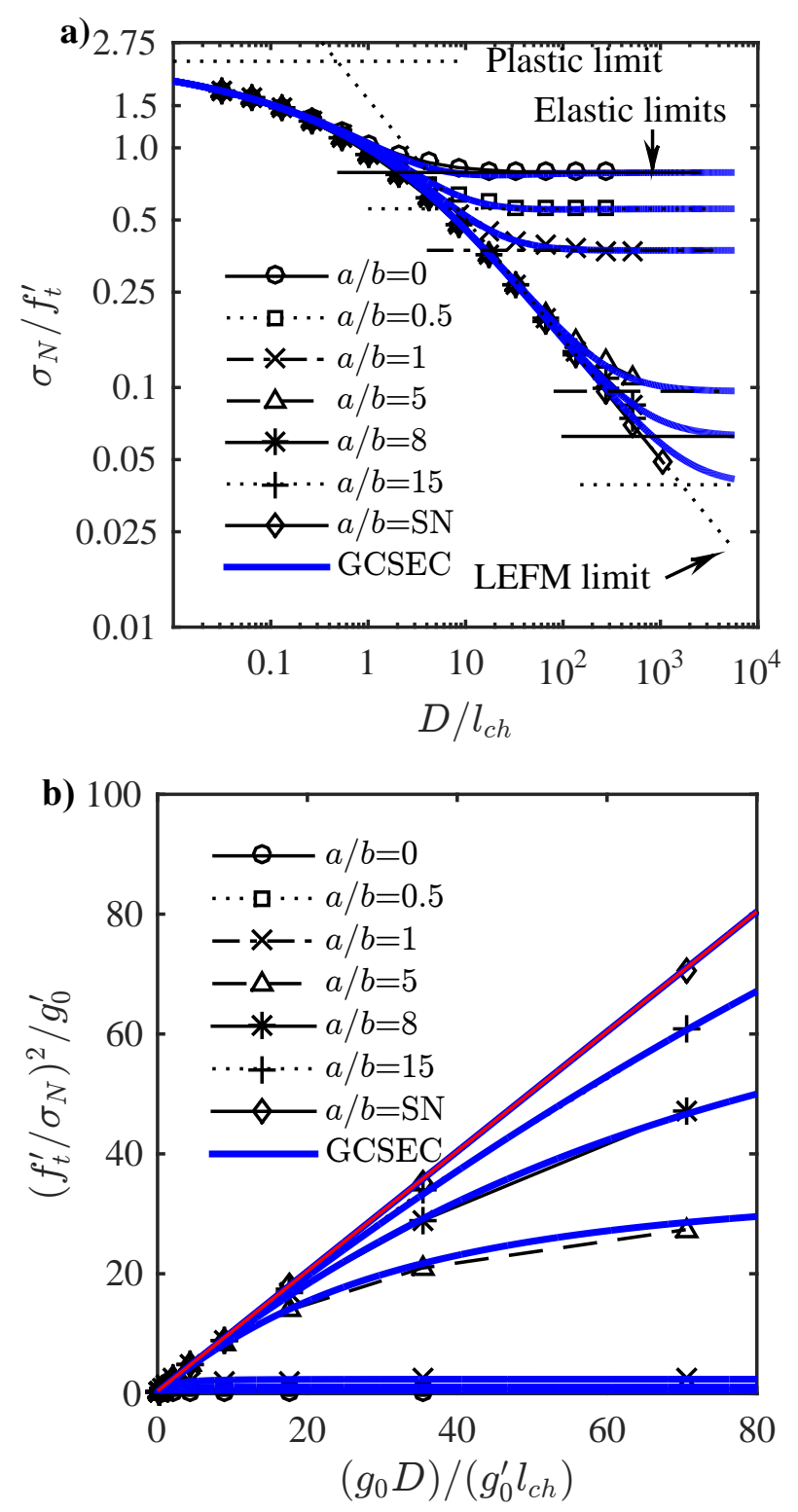

Figure 5: TPB Configuration: a) size effect curves for elliptically notched geometries; b) slope of size effect curves versus normalized size; c) slope of size effect curves versus normalized width of elliptical notch; d) size effect curve for constant-width notch geometry.

It is worth noting that both the size effect of type I and II due to the cohesive crack law tends, for $D \rightarrow 0$, to the same value, i.e. same plastic (or strength) limit. As it can be seen from Figs 4 and 5 for $\alpha=0.125$ the size effect behavior is always the same for $D / l_{c h}<1$. However, when $\alpha \rightarrow 0$ or $a / b \rightarrow 0$ the normalized energy release rate $g_{0}$ is equal to zero. As for the blunt notch, also in this case the normalized energy release rate, $g_{0}$, and its derivative, $g_{0}^{\prime}$, in Eq. 8 are obtained for the sharp notch case with the given $\alpha$ and for $a / b \rightarrow 0$. In any case the GCSEC in Eq. 8 gives the correct asymptotic behavior also for no notch structure. In fact for $g_{0} \rightarrow 0$ one gets

$$
\begin{aligned}
\sigma_{N u}=f_{t}^{\prime}\left(g_{0} \frac{D}{l_{c h}}+\frac{1}{\beta_{p}^{2}}\right)^{-1 / 2} & \left(g_{0} \beta_{e}^{2} \frac{D}{l_{c h}}+1\right)^{1 / 2}
\end{aligned}
$$

which for $D \rightarrow 0$ and for $D \rightarrow \infty$ gives $\sigma_{N}=f_{t}^{\prime} \beta_{p}^{2}$ and $\sigma_{N}=f_{t}^{\prime} \beta_{e}$, respectively, i.e. the plastic and elastic limit.

\section{CONCLUSIONS}

The size dependence of structural strength has been studied using a linear cohesive crack law and considering the effect of different types of notch: blunt and sharp. This work significantly extends the results of a previous study [1] because it studies the size effect behavior of the three-point bending with span/depth ratio equal to 3 and of the center crack panel with an elliptical blunt notch with constant aspect ratio to preserve geometric similarity.

The following main conclusions can be drawn

1. For specimens with blunt notches, the cohesive size effect curves are characterized by a continuous transition from the plastic behavior for small sizes to the purely brittle behavior with failure at crack initiation for large sizes. Hence, these size effect curves tend asymptotically to the elastic limit.

2. For specimens with blunt notches, in a double logarithmic plot, the cohesive size effect curves feature a point of inflection where the curve curvatures transition from negative to positive. At this inflection point the curve slope approaches the 
LEFM slope of $-1 / 2$ for increasing stress concentration at the notch tip. The point of inflection occurs when the notch width is approximately equal to twice the material characteristic length, $2 l_{c h}$.

3. In the case of sharp notches, for which the elastic limit is zero, the elastic asymptote is located, theoretically, at $-\infty$ in a double logarithmic plot. In this condition the size effect curve does not have an inflection point and it approaches asymptotically the LEFM limit.

4. For sharp notches, the crack stress profiles at the peak load for different sizes reveal that the FPZ length converges to constant a value of $0.71 l_{c h}$ (for linear softening) and the and notch tip cohesive stress converges to zero as the structural size increases. For blunt notches, however, the behavior is completely different. In fact, at the peak load the length of the FPZ first increases and then it shrinks for increasing structural size. Asymptotically, it becomes a mathematical point and the stress at the notch tip tends to the tensile strength, $f_{t}^{\prime}$. As a consequence, for specimens with blunt notch and contrarily to the case of sharp notches, the size of the FPZ at peak load is not a material property.

5. The numerical results on structural strength can be approximated accurately with an analytical equation, entitled Generalized Cohesive Size Effect Curve (GCSEC), which is valid for different structural configurations with both blunt or sharp notch. The GCSEC is able to describe both the type I and type II size effect.

6. The GCSEC depends on the cohesive crack parameters such as the tensile strength, $f_{t}^{\prime}$, the fracture energy, $G_{f}$, and, as a consequence, the characteristic length, $l_{c h}$. The GCSEC takes into account the effect of different structural configurations through the LEFM nondimensional energy release rate and its derivative.

\section{REFERENCES}

[1] Cusatis, G., Schauffert, E.A., 2009. Cohesive crack analysis of size effect. Engineering Fracture Mechanics 76:21632173.

[2] Hillerborg, A., Mooder, M., Petersson, P.E., 1976 Analysis of crack formation and crack growth in concrete by means of fracture mechanics and finite elements. Cement and Concrete Research 6:773782.

[3] Planas J., Guinea G.V., Elices M., 1997.Generalized size effect equation for quasi-brittle materials. Fatigue \& Fracture of Engineering Materials \& Structures 20(5):671-687.

[4] Bažant, Z.P. and Planas, J., 1998. Fracture and size effect in concrete and other quasibrittle materials. Boca Raton and London: CRC Press.

[5] Bažant, Z.P., and Kazemi, M.T. 1990. Determination of fracture energy, process zone length and brittleness number from size effect, with application to rock and concrete. International Journal of Fracture 44(2): 111-131.

[6] Labuz, J.F. and Biolzi, L. 1998. Characteristic strength of quasi-brittle materials, International Journal of Solids and Structures 35(31-32):4191-4203.

[7] Cedolin, L. and Cusatis, G. 2008. Identification of concrete fracture parameters through size effect experiments, Cement and Concrete Composites 30(9):788-797.

[8] Di Luzio, G., and Cusatis, G. 2018. Cohesive crack analysis of size effect for 
samples with blunt notches and generalized size effect curve for quasi-brittle materials. Engineering Fracture Mechanics 204:15-28.

[9] Hughes, T.J.R., 2000. The finite element method: linear static and dynamic $f_{i-}$ nite element analysis. Mineola, New York: Dover Publications.

[10] Bfer, G., 1985. An isoparametric joint/interface element for finite element analysis. International Journal for Numerical Methods in Engineering 21(4):585-600.

[11] Young, W. C., Richard G. B., 2002. Roark's Formulas for Stress and Strain.
Seventh Edition. McGraw-Hill.

[12] Timoshenko, S. P., Goodier, J. N., 1970. Theory of elasticity. McGraw-Hill, New York.

[13] Planas, J., Guinea, G.V., Elices, M., 1995. Rupture modulus and fracture properties of concrete. In F. H. Wittmann (ed), Fracture Mechanics of Concrete Structures, Vol. I, pp. 95-110. Aedificatio Publishers, Freiburg, Germany.

[14] Bažant, Z.P., Yu, Q., Zi, G., 2002. Choice of standard fracture test for concrete and its statistical evaluation. International Journal of Fracture 118:303-337. 